\title{
An On Body Accelerometer System for streaming therapy data using COTS UHF RFID
}

\author{
Robert Horne \\ School of Engineering and Digital Arts \\ University of Kent \\ Canterbury, United Kingdom \\ r.j.horne@kent.ac.uk \\ John Batchelor \\ School of Engineering and Digital Arts \\ University of Kent \\ Canterbury, United Kingdom \\ j.c.batchelor@kent.ac.uk
}

\author{
Philippa Jones \\ School of Engineering \\ Cardiff University \\ Cardiff, United Kingdom \\ jonesp29@ cardiff.ac.uk \\ Catherine Holt \\ School of Engineering \\ Cardiff University \\ Cardiff, United Kingdom \\ holt@ cardiff.ac.uk
}

\author{
Paul Taylor \\ School of Engineering and Digital Arts \\ University of Kent \\ Canterbury, United Kingdom \\ P.Taylor-250@kent.ac.uk
}

This paper details the use of an on body Accelerometer COTS RFID system for use in determining specific therapeutic movements, and the performance of said movements. Conventional methodologies rely on power intensive Bluetooth or Wi-Fi technologies for live communications, but this paper outlines the use of ultra-low power battery assistive passive modes on UHF RFID chips to harness sub$2 m W$ continuous streaming of Accelerometer data.

Keywords-UHF, RFID, Accelerometer, low-power, COTS

\section{INTRODUCTION}

Over 1 million osteoarthritis (OA) related total knee replacement (TKR) surgeries take place across the UK annually [1], with patients undergoing regular post-surgery physiotherapy that is reliant on home-based exercise rehabilitation [2] and driven by personalized self-management. With poor patient adherence that is difficult to ascertain [3], clinicians who are challenged to optimize patient outcomes are unable to determine whether improvements (or lack of) can be attributed to an exercise intervention or (non) adherence. There is a clear need for enhanced forms of objectively monitoring patient adherence to homebased exercise rehabilitation, providing valuable biomechanical knowledge to clinicians to guide personalized exercise prescription. This could provide rigorous adherence measurements, optimize the rehabilitation process, reduce NHS burden and improve patient satisfaction.

The potential applications of body-worn sensors to monitor physical activity and rehabilitation is well recognised, with a number of research groups investigating the optimal sensing platform for objectively detecting the performance of rehabilitation exercises $[4,5]$. Some of the known design considerations for these platforms include the need for low power consumption and transmission power [6], a light device that is comfortable to wear that has a low profile [7] and one that is cost effective for successful integration within the NHS framework [8].

This paper introduces a new methodology in the form of using UHF RFID instead of the standard norm of Bluetooth or WiFi sensor platforms. The reason for choosing this communication methodology is due to its inherent low power communications functionality, as well as low implementation cost.

\section{SYSTEM DESIGN}

The system detailed in this paper can be broken down into several critical areas.

\section{A. Acceleration Measurement}

The acceleration measurement was taken by an ADXL362 [9] at $100 \mathrm{~Hz}$, which was transmitted to the microcontroller via SPI and interrupt interface. This device was chosen due to its low power characteristics, package size and ability to use interrupts to enable a FIFO read. Within the lowest power state, the ADXL consumes $270 \mathrm{nA}$ and only $2 \mu \mathrm{A}$ at $100 \mathrm{~Hz}$. The ADXL362 reports axis acceleration as a 12 bit value, however when processed, four additional padding bits are attached to result in a 16 bit value.

\section{B. Embedded Measurement}

To process and forward the incoming data from the ADXL362 Accelerometer an ATSAMD21G [10] microcontroller from Atmel was used. This microcontroller was chosen due to its ultra-low power characteristics and general availability. The microcontroller is required to modify the incoming data from the accelerometer into a form that is applicable for the volatile memory on the RFID chip. Using interrupts from both the ADXL362 and the RFID chip, the microcontroller can remain in low power states (sub $10 \mu \mathrm{A}$ ) for the majority of the time that the device is active, thus lowering overall power consumption. The microcontroller could be programmed via the 10-pin breakout port or via the USB socket utilising a bootloader. The inclusion of USB also allowed for debugging via a serial connection to the PC using Putty. The estimated cost to produce the entire embedded module is roughly $£ 10$. 


\section{UHF RFID Communications}

The RFID UHF chip used was a commercially off the shelf (COTS) EM4325 [11] made by EM Microelectronic transmitting to a Jadak ThingMagic M6E reader. The Jadak reader has a cost of roughly $£ 1000$. The EM4325 was chosen due to its RAM register access through SPI. The majority of commercially available UHF RFID chips have memory access as a standard feature, but this is typically access to EEPROM. EEPROM is too slow for the transfer speeds required for $60 \mathrm{~Hz}$ accelerometer streaming and poses the issue that it has a low amount of lifetime cycles. The EM4325 also has the ability to change its transmission characteristics dependent on whether it has an external power supply. This "battery assistive passive" mode enables an extension in read range and memory access speeds. A wire dipole antenna was implemented onto the printed circuit board.

\section{Software side analysis}

To enable communications to the Jadak ThingMagic M6E reader [12], an Application Programming Interface (API) from Jadak was used. The API allowed for a software interface written in C\# to be implemented to control the flow of data from the ThingMagic reader. The software interface enabled the implementation of commands specific to the EM4325's volatile memory and for the higher backscatter frequency required to achieve the desired data transfer rate of 960 bytes per second ( 16 bytes sent at $60 \mathrm{~Hz}$ ). To ensure data integrity a frame number and checksum were sent with the accelerometer data (Fig.1.). A reserved section was implemented for further development of the platform to include extra sensor data.

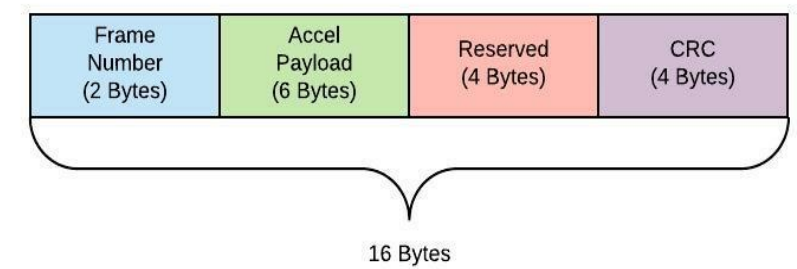

Fig. 1. - RFID Register Memory Packet Structure

When the sensor data is collected by the PC, it generates an internal Unix timestamp and computes whether the frame is valid based on the cyclic redundancy check (CRC) and payload provided. As the connection to the reader is done via a com port, threading is used to negate any latency that may arise. If the frame is valid, it is added to a buffer, if it is invalid, the data is invalidated but reference of the frame is still kept. Once the buffer has been filled, the contents are stored into a comma separated value (CSV) file for offline processing in Excel or Matlab. From there, update rate can be inferred from the number of samples and the duration between timestamps. The acceleration vectors are easily extracted from the stored payload, as they have their own columns within the file. Other metrics from the read of the RFID Memory are also logged such as the RSSI, Phase and frequency. To ensure that when logging data that only the specific RFID Tag is communicating with the system, an EPC filter was implemented, and each of the RFID tags were given a unique identifier with the first four characters having the same identifier.

\section{E. Power Management}

To enable the device to function, a lithium polymer (LIPO) battery was attached to the printed circuit board. The battery had a capacity of $120 \mathrm{mAh}$ which was more than sufficient for the entire duration of a clinical session. Active current draw was measured in the region of $500 \mu \mathrm{A}$ with the input voltage mirroring a standard LIPO at 3.7V. The LIPO was chosen as it matched the size of the printed circuit board. This battery could be recharged using an integrated LIPO charger. The charger could be activated if a USB connection was made to a computer or a suitable 5V source power supply. The charger was limited to a charge rate of 50mA to ensure the longevity of the battery over multiple charging cycles.

The complete system block diagram can be seen in Fig. 2, and the rigid prototype printed circuit board can be seen in Fig. 3 . 


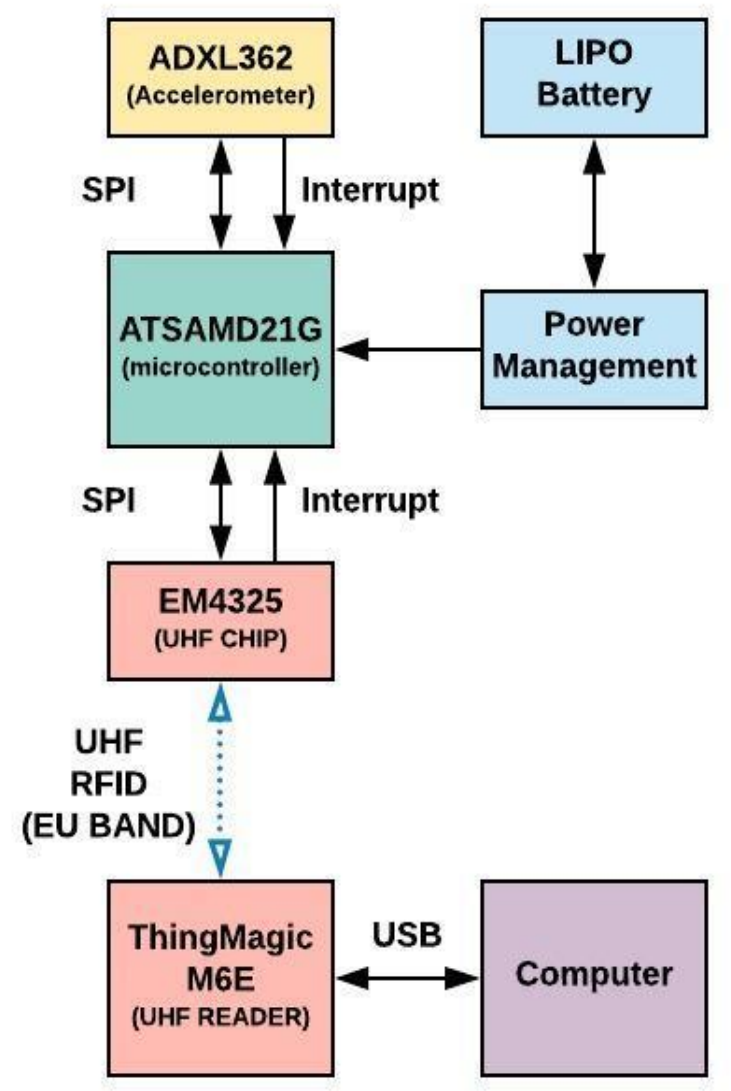

Fig. 2. - System Block Diagram

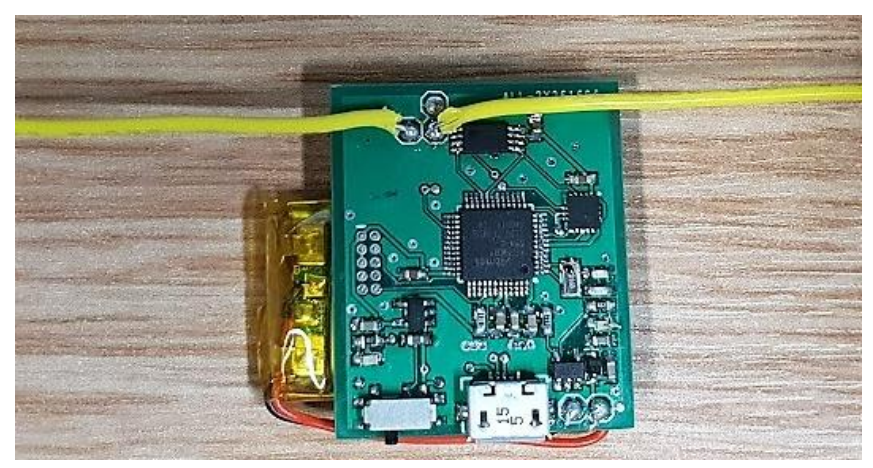

Fig. 3. - Rigid Prototype UHF Accelerometer Streaming Board

\section{TESTING PROTOCOL}

In order to validate the performance and reliability, a gold standard inertial sensor system was used in parallel with the UHF RFID system. To collect the data, the Musculoskeletal Biomechanics Research Facility within the School of Engineering at Cardiff University was used. The gold standard system chosen was the Xsens MVN. This was chosen because of its common use within the academic field, accuracy and communication system, which did not interfere with the system being tested. The Xsens system starts at a price of roughly $€ 3,000[13]$.

The Xsens MVN Software uses a specific sensor unit called the MTw sensor; it can be seen in Fig.4 and Fig.5 as the orange boxed device located on a black Velcro strap. 


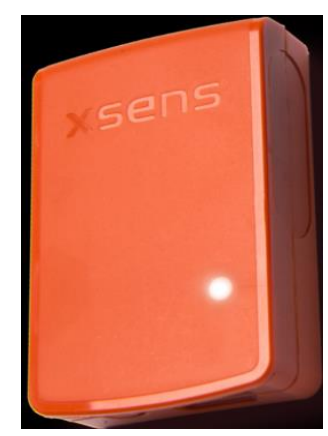

Fig. 4. - Xsens Accelerometer unit

The devices in use were located on the Right Thigh and Right Shank region. Other locations can be used such as the foot, but for this experiment, only two locations were sampled via the proposed system and the lower body Xsens sensor set was utilized ( 7 sensors - pelvis and bilateral thigh, shank and feet) to enable comparator sensors for RFID devices placed on the right thigh and shank.

To try to get a reliable correlation between the two systems, the RFID and Xsens sensor units were placed as close as possible to each other on the body as can be seen in Fig. 5. This allowed for a direct comparison of the data being produced by both sensor units in an offline domain.
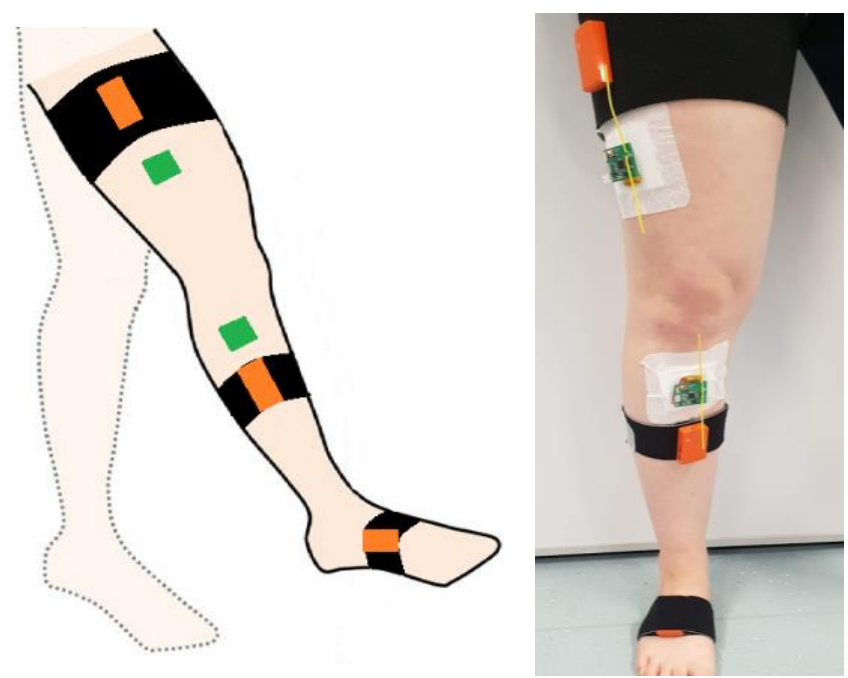

Fig. 5. - Sensor locations for both Xsens and RFID units

To establish a baseline of acceleration performance, five specific movements were chosen to illustrate representative patient therapy actions:

- KEX - Knee Extension

- $\quad$ KFL - Knee Flexion

- $\quad$ SLB - Single Leg Balance

- $\quad$ STS - Sit to Stand

- WSH - Weight Shifting

These five early phase knee rehabilitation exercises are included in the Taxonomy for Rehabilitation of knee conditions [14] and are routinely prescribed to those with knee pain or following surgical intentions such as a total knee replacement.

Each of the specific actions were repeated 5 times, with 5 repetitions within the action. This resulted in a test data set of 25 unique data samples for each experiment. The experiment was conducted two times, due to requiring both Right Shank and Right Thigh data capture. The UHF RFID reader was placed 1.5 meters from the subject during each test. The range 
performance of the tag did indicate that more range could be achieved, but a lower value was chosen to ensure the best likelihood of packet integrity.

Each of the RFID sensors were attached to plasters using high strength double-sided tape. Both Xsens units were mounted using elasticated straps.

\section{RESULTS}

To provide an assessment, each of the data sets were compared, looking at the specific graphical signatures produced from each repetition. It was observed that the correlation between the RFID generated and the Xsens Acceleration Vector data was visually high. Giving a clear indication that specific movements, with different acceleration patterns could be isolated from both the RFID and Xsens data set, as well as having a clear correlation between RFID and the Xsens gold standard data.

For reference, a sample Knee Flexion (Shank Focus), exercise can be seen in Fig.6, which illustrates the outputted graphical waveforms generated from each system, with the black waveforms being the RFID Sensor data, and the blue being the Xsens gold standard system under test.

Regarding X axis acceleration, it can be seen in Fig. 6 that both systems produce a clear oscillation of five repetitions of a Knee Flexion in respect to acceleration, both having significant visual similarities regarding peaks and troughs. The minor differences seen can be attributed to timing inconstancies on the RFID communications side causing a larger state of change. Nevertheless, each of the five repetitions can be clearly identified. The initial Acceleration vector can also be seen to be different on the two devices, this can be attributed to the sensors having slightly different resting orientations.
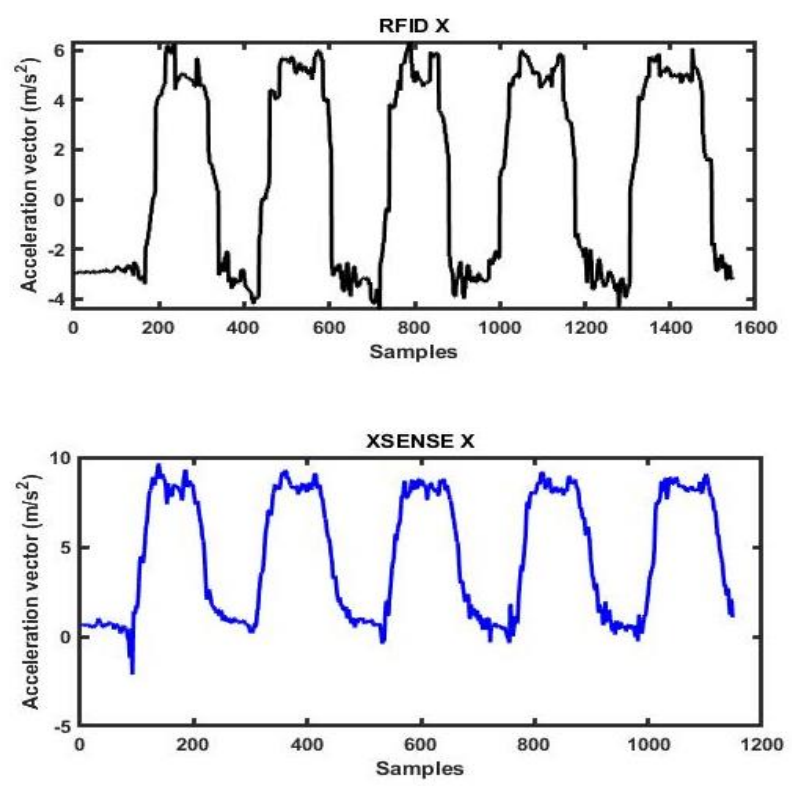

Fig. 6. - Accelerometer Data X Axis

In the Y Axis Fig.7, a slight variation is observed; however, each repetition is still evident. One of the issues theorized for this lack of clarity in replication of the waveform could be due to the specific mounting on the RFID sensor. With the extra height generated from the LIPO battery and multiple layers of adhesive tape, the translation of acceleration force could have been dampened. 

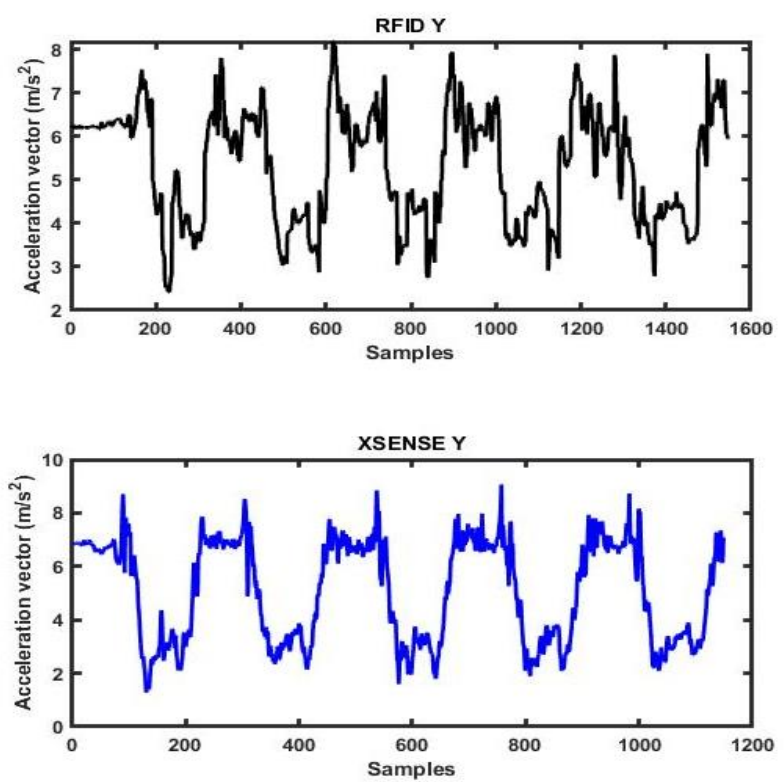

Fig. 7. - Accelerometer Data Y Axis

A small variation can be seen in the Z Axis in Fig. 8. Again, the movements can be determined with the RFID sensor but with less feature data apparent. A probable cause for this lack of feature data, can again be attributed to the initial mounting methodology used. It should be noted that the specific Knee Flexion (Shank Focus) exercise did not produce large acceleration values in the $\mathrm{Z}$ Axis, though the measured acceleration range is in line with the $\mathrm{X}$ and $\mathrm{Y}$ Axis movement.
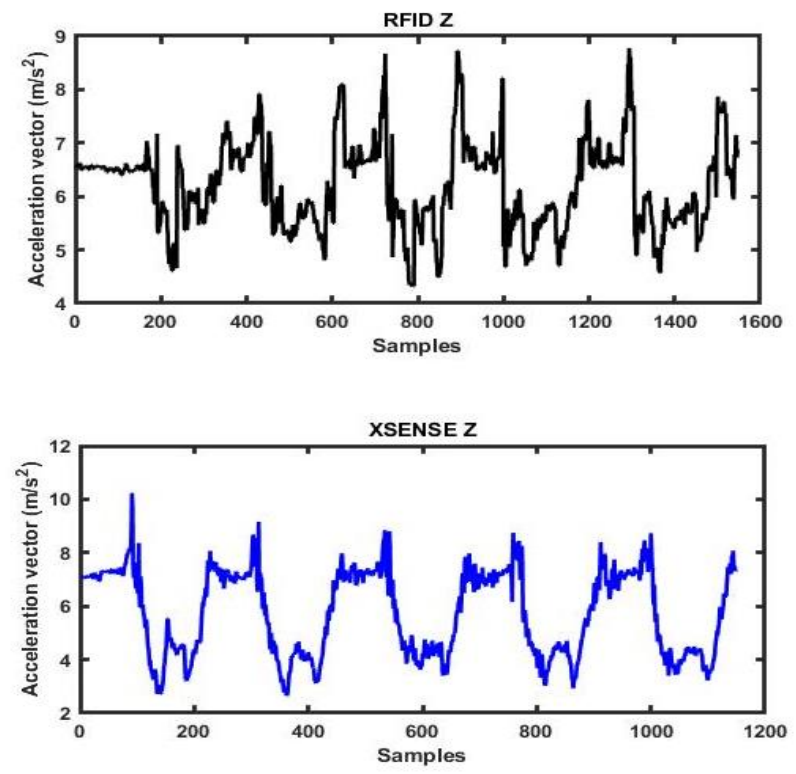

Fig. 8. - Accelerometer Data Z Axis

The unit has been tested for durability in streaming performance, to ensure it does not have the same degradation and failure that EEPROM based streaming incurs. The device after a testing period of 3-5 hours a day for 2 months is still at $100 \%$ performance. As the register memory of the EM4325 is used instead of EEPROM, it is theorised that the lifetime of the device will be battery dependant. This is a major improvement over the EEPROM lifecycle of 100,000 writes, which would equate to 30 minutes of constant operation.

The power consumption of the device being within the region of $500 \mu \mathrm{A}$ during loading, gave an estimated on-time of roughly 240 hours, and more than 1000 hours in standby. 


\section{CONCLUSION AND FURTHER WORK}

To conclude, the paper outlines the use of an ultra-low power UHF RFID accelerometer streaming system, for the use of analyzing the adherence to specific therapeutic movements. The results show a clear correlation with the gold standard currently being used in a clinical setting. It can be seen using a lower cost system, though it does currently have some minor resolution inconsistencies, but has the potential to match the gold standard. Subsequently the power consumption of the UHF RFID system could potentially provide sufficient longevity to last for 1 month of clinical trials during work hours without the need to be charged.

Further work will include increasing the speed and resolution of data being generated by the RFID Sensor device. Additional testing procedures will be developed to enable orientation syncing to allow for higher levels of correlation to be achieved. As mentioned, extra space in the register memory will be utilized to support an exploration into adding other sensors such as Electrocardiogram (ECG). Furthermore, the printed circuit board will be migrated to a flexible substrate such as Kapton or Mylar for better adhesion to the target body area to reduce the dampening effects, which were found to affect specific axes during the movements.

\section{ACKNOWLEDGMENT}

This research was supported by the EPSRC projects Adaptive Assistive Rehabilitative Technology: Beyond the Clinic (AARTBC) (EP/M025543/1) and Formulating and Manufacturing Low Profile Integrated Batteries for Wireless Sensing Labels $(\mathrm{EP} / \mathrm{R} 02331 \mathrm{X} / 1)$

\section{REFERENCES}

[1] National Joint Registry, 2018

[2] Holden MA1, Nicholls EE, Hay EM, Foster NE. (2008). Physical therapists' use of therapeutic exercise for patients with clinical knee osteoarthritis in the United Kingdom: in line with current recommendations? Physical Therapy. Oct; 88(10):1109-21. doi: 10.2522/ptj.20080077. Epub 2008 Aug 14.

[3] Forkan R1, Pumper B, Smyth N, Wirkkala H, Ciol MA, Shumway-Cook A. Exercise adherence following physical therapy intervention in older adults with impaired balance. Physical Therapy. 2006 Mar;86(3):401-10.

[4] Portia E. Taylor, Gustavo J.M. Almeida, Takeo Kanade and Jessica K. Hodgins (2010). Classifying Human Motion Quality for Knee Osteoarthritis Using Accelerometers, Portia. 32nd Annual International Conference of the IEEE EMBS Buenos Aires, Argentina, August 31 - September 4.

[5] Kun-Hui Chen, Po-Chao Chen, Kai-Chun Liu and Chia-Tai Chan (2015) Wearable Sensor-Based Rehabilitation Exercise Assessment for Knee Osteoarthritis. Sensors, 15, 4193-4211; doi:10.3390/s150204193

[6] Alemdar, H., and Ersoy, C. (2010). Wireless sensor networks for healthcare: A survey. Computer Networks. Volume 54, Issue 15, 28, Pages $2688-2710$.

[7] Papi, E., Belsi, A. and McGregor, A.H. (2015). A knee monitoring device and the preferences of patients living with osteoarthritis: a qualitative study, BMJ Open, 5:e007980.

[8] Papi E, Murtagh GM, McGregor AH. Wearable technologies in osteoarthritis: a qualitative study of clinicians’ preferences. BMJ Open 2016;6:e009544. doi:10.1136/bmjopen-2015- 009544

[9] Analog.com, 2019. [Online]. Available: https://www.analog.com/media/en/technical-documentation/data-sheets/adxl362.pdf. [Accessed: 20- Jan- 2019]

[10] ww1.microchip.com, 2019. [Online]. Available: http://ww1.microchip.com/downloads/en/DeviceDoc/SAMD21-Family-DataSheet-DS40001882D.pdf. [Accessed: 20- Jan- 2019]

[11] Emmicroelectronic.com, 2019. [Online]. Available: https://www.emmicroelectronic.com/sites/default/files/products/datasheets/4325-ds_0.pdf. [Accessed: 20- Jan- 2019]

[12] Jadaktech.com, 2019. [Online]. Available: https://www.jadaktech.com/products/rfid/embedded-uhf-rfid-readers/mercury6e-m6e/. [Accessed: 20- Jan2019]

[13] Xsens.com 2019 [Online]. Available: https://shop.xsens.com/shop/mtw-awinda/mtw-awinda-bundles/mtw-awinda-research-bundle/mtw-awindaresearch-bundle-mtw2-dk-2 I

[14] Button, Kate, Van Deursen, Robert William Martin, Soldatova, Larisa and Spasic, Irena 2013. TRAK ontology: defining standard care for the rehabilitation of knee conditions. Journal of Biomedical Informatics 46 (4) , pp. 615-625. 10.1016/j.jbi.2013.04.009 